\title{
Measured Local Heat Transport in Turbulent Rayleigh-Bénard Convection
}

\author{
X.-D. Shang, ${ }^{1}$ X.-L. Qiu, ${ }^{2}$ P. Tong, ${ }^{2}$ and K.-Q. Xia ${ }^{1}$ \\ ${ }^{1}$ Department of Physics, The Chinese University of Hong Kong, Shatin, Hong Kong, China \\ ${ }^{2}$ Department of Physics, Oklahoma State University, Stillwater, Oklahoma 74078
}

(Received 19 September 2002; published 20 February 2003)

\begin{abstract}
Local convective heat flux in turbulent thermal convection is obtained from simultaneous velocity and temperature measurements in an aspect-ratio-one convection cell filled with water. It is found that fluctuations of the vertical heat flux are highly intermittent and are determined primarily by the thermal plumes in the system. The experiment reveals a unique mechanism for the heat transport in turbulent convection.
\end{abstract}

DOI: 10.1103/PhysRevLett.90.074501

PACS numbers: 47.27.Te, 44.25.+f

An important issue in the study of turbulent RayleighBénard convection is to understand how heat is transported vertically through a convection cell [1-3]. A large number of global heat transport measurements have been carried out in various convecting fluids and under different experimental conditions. Some of the measurements were conducted with wide parameter range and great precession [4-9]. These measurements have stimulated considerable theoretical efforts, aimed at explaining the functional form of the measured Nusselt number (normalized heat flux), $\mathrm{Nu}(\mathrm{Ra}, \mathrm{Pr})$, as a function of the two experimental control parameters: the Rayleigh number $\mathrm{Ra}$ and the Prandtl number Pr. Like many transport phenomena in condensed matter physics, the measured macroscopic transport properties can often be explained by theories with different microscopic mechanisms [1-4]. A main issue of an unresolved theoretical debate is whether the heat transport in turbulent convection is determined primarily by thermal plumes, which erupt from the upper and lower thermal boundary layers, or by the large-scale circulation (LSC) that spans the height of the convection cell. Direct measurements of the local convective heat flux, therefore, become essential to the understanding of the heat transport mechanism in turbulent convection.

In this Letter, we report direct measurements of the normalized local convective heat flux, $\mathbf{J}(\mathbf{r})=$ $\langle\mathbf{v}(\mathbf{r}, t) \delta T(\mathbf{r}, t)\rangle_{t} H / \kappa \Delta T$, over varying Rayleigh numbers and spatial positions $\mathbf{r}$ across the entire cell. Here $\kappa$ is the thermal diffusivity of the convecting fluid, $\Delta T$ is the temperature difference across the cell height $H(=20.5 \mathrm{~cm})$, and $\langle\ldots\rangle_{t}$ represents an average over time $t$. In the experiment, the local temperature fluctuation $\delta T(\mathbf{r}, t)=T(\mathbf{r}, t)-$ $T_{0}$ and the flow velocity $\mathbf{v}(\mathbf{r}, t)$ are measured simultaneously. The mean temperature $T_{0}$ of the bulk fluid is kept at $\sim 30^{\circ} \mathrm{C}$ and the corresponding Prandtl number, $\operatorname{Pr}=\nu / \kappa$, is $\sim 5.4$. The convection cell is an upright cylindrical cell of aspect ratio one and is filled with water. Details about the apparatus have been described elsewhere[10]. The upper and lower plates are made of brass and the sidewall is a transparent Plexiglas ring with a narrow and long rectangular flat window for velocity measurement. Two silicon rubber film heaters connected in parallel are sandwiched on the backside of the lower plate to provide constant and uniform heating. The upper plate is in contact with a cooling chamber, whose temperature is maintained constant by circulating cold water from a temperature bath.

Local velocity measurements are conducted using a laser Doppler velocimetry (LDV) system together with an argon-ion laser[11]. The LDV system can simultaneously measure two components of $\mathbf{v}(\mathbf{r}, t)$ as a function of time $t$ and the measuring position $\mathbf{r}$ (laser focusing spot) is varied by moving the LDV fiber-optic transceiver probe, which is mounted on a traversable table. It has been shown[11] that LDV is capable of measuring the flow velocity with high accuracy (better than $1 \%$ ) over the entire convection cell, except near the upper and lower thermal boundary layers, whose thickness is $\sim 1 \mathrm{~mm}$. The sampling rate of the velocity measurements is $\sim 15 \mathrm{~Hz}$, which is much larger than the cutoff frequency of the velocity power spectrum. Typically, we take two to seven hour-long time series data $\left(\sim 5 \times 10^{5}\right.$ data points $)$ at each location, ensuring that the statistical average of the flow properties is adequate.

Simultaneous velocity and temperature measurements are carried out using a multichannel LDV interface module to synchronize the data acquisition. A triggering pulse from the LDV signal processor initiates the acquisition of an analog temperature signal. A small movable thermistor of $0.2 \mathrm{~mm}$ in diameter, $15 \mathrm{~ms}$ in time constant, and $1 \mathrm{mK} / \Omega$ in temperature sensitivity is used to measure $T(\mathbf{r}, t)$. To guide the thermistor into the cell, we install a horizontal stainless steel tube of $1.1 \mathrm{~mm}$ in diameter at the midheight of the sidewall. The tube can slide in and out so that $T(\mathbf{r}, t)$ can be measured at various horizontal positions along a cell diameter ( $x$ axis) from the sidewall $(x / H=0)$ to the cell center $(x / H=0.5)$. Similarly, we also install a vertical stainless steel tube through the center of the top plate and measure $T(\mathbf{r}, t)$ along the central vertical axis of the cell ( $z$ axis) from the bottom $(z / H=0)$ to the cell center $(z / H=0.5)$. In the experiment, the spatial separation between the LDV 
focusing spot and the thermistor tip is kept at a minimal value of $0.7 \pm 0.2 \mathrm{~mm}$. This distance is 3 times larger than the tip diameter of the thermistor but 20 times smaller than the correlation length between the temperature and velocity fluctuations [12]. In addition, the LDV focusing spot is always placed at an upstream position to further minimize the disturbance of the thermistor to the velocity measurement.

We first discuss the measurements of $\mathbf{J}(\mathbf{r})$ along the $x$ and $z$ axes; both are in the rotation plane of LSC. For the horizontal scan, we measure the vertical heat flux $J_{z}(x)$ and the horizontal heat flux $J_{y}(x)$ (out of the rotation plane). For the vertical scan, we measure the horizontal heat flux $J_{x}(z)$ and the vertical heat flux $J_{z}(z)$. Figure 1(a) shows the measured $J_{z}(x)$ (circles) and $J_{y}(x)$ (triangles) as a function of the normalized horizontal position $x / H$ at two different values of Ra. It is seen that the heat flux across the midheight plane of the cell is predominately in the vertical direction and the horizontal heat flux is

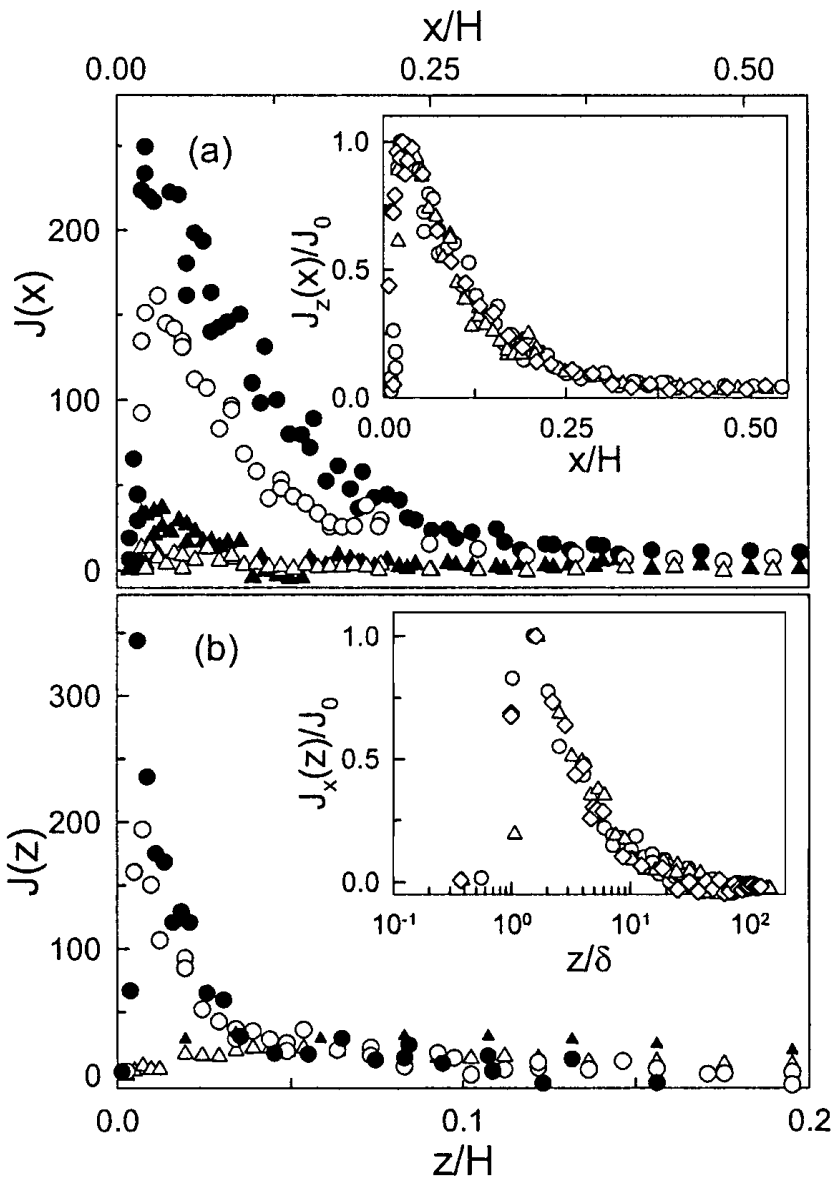

FIG. 1. (a) Measured vertical flux $J_{z}(x)$ (circles) and horizontal flux $J_{y}(x)$ (triangles) as a function of $x / H$. (b) Measured horizontal flux $J_{x}(z)$ (circles) and vertical flux $J_{z}(z)$ (triangles) as a function of $z / H$. The measurements in (a) and (b) are made at $\mathrm{Ra}=1.8 \times 10^{9}$ (open symbols) and $6.0 \times 10^{9}$ (closed symbols). The two insets show, respectively, the normalized $J_{z}(x) / J_{0}$ and $J_{x}(z) / J_{0}$ at $\mathrm{Ra}=1.8 \times 10^{9}$ (triangles), $3.6 \times 10^{9}$ (diamonds), and $6.0 \times 10^{9}$ (circles). negligible. The vertical heat flux concentrates in the sidewall region and increases with Ra. While the amplitude of the flux profile $J_{z}(x)$ increases with $\mathrm{Ra}$, its shape remains invariant with Ra. This is clearly shown in the inset, which displays the normalized $J_{z}(x)$ by its peak value $J_{0}$ at three different values of Ra. The vertical heat flux at the cell center is small but is definitely nonzero. It accounts for $\sim 5 \%$ when compared with the peak value $J_{0}$ near the sidewall.

Figure 1(b) shows the measured $J_{x}(z)$ (circles) and $J_{z}(z)$ (triangles) as a function of the normalized vertical position $z / H$ at two values of Ra. In contrast to the situation near the sidewall, heat transport near the center of the lower conducting surface is dominated by the horizontal flux along the direction of LSC and the vertical flux is negligible. When compared with Fig. 1(a), we find that $J_{x}(z)$ peaks at a location much closer to the lower boundary and the width of the peak is narrower. The amplitude of the flux profile $J_{x}(z)$ increases with Ra, but its shape remains unchanged with $\mathrm{Ra}$ (see the inset). Another important feature shown in the inset is that the peak position of $J_{x}(z)$ scales with the thermal boundary layer thickness $\delta\left(=425 \mathrm{Ra}^{-0.285} \mathrm{~mm}\right.$ [13]). Figure 1 thus reveals that heat transport in the aspect-ratio-one cell is carried out mainly along the cell periphery in the direction of LSC.

Our recent temperature and velocity measurements $[11,14]$ showed that the spatial distribution of thermal plumes in a closed cell is neither homogeneous nor isotropic. The thermal plumes organize themselves in such a way that warm plumes accumulate on one side of the cell and cold plumes concentrate on the opposite side of the cell. Figure 2 shows the plume distribution with warm rising plumes on the left and cold falling plumes on the right [15]. The warm and cold plumes, which are separated laterally in the two opposing sidewall regions, exert buoyancy forces on the fluid and drive the vertical flow near the sidewall. The central core region is "sheared" by the rising and falling plumes, resulting in a large-scale circulation across the cell height [11].

From these measurements we conclude that the dynamics in turbulent convection is determined primarily by the thermal plumes. The spatial separation of warm and cold plumes and the resulting LSC provide a fast channel along the cell periphery for the transport of heat. This physical picture explains our finding that the plumedominated sidewall region (of thickness $\sim H / 4$ ) coincides with the "peak region," in which both $J_{z}(x)$ and $v_{z}$ reach maximum. Clearly, this is a self-organizing process in that the plume separation and LSC help each other and they are not independent anymore. Because velocity fluctuations in the central region are strong, most thermal plumes are mixed up in the region. Nevertheless, there are still some unmixed warm and cold plumes remaining, which give rise to a nonzero $J_{z}$ in the region.

Besides the spatial organization, the warm and cold plumes are also coupled in time. As shown in Fig. 3(a), 


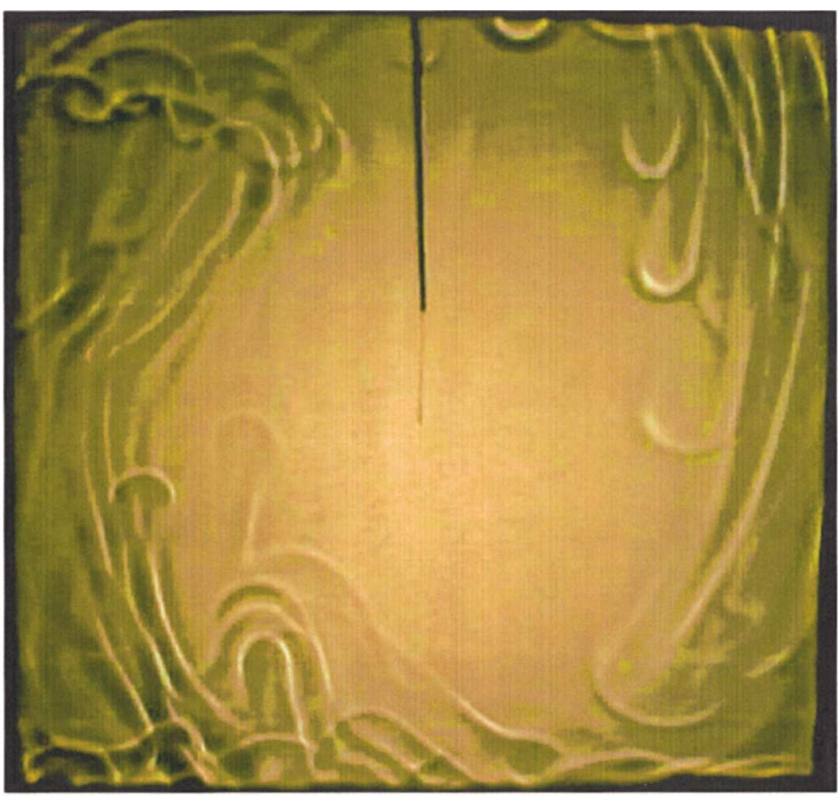

FIG. 2 (color). A shadowgraph showing the spatial distribution of thermal plumes in an aspect-ratio-one cell $(H=15 \mathrm{~cm})$ filled with dipropylene glycol $(\operatorname{Pr}=596)$. The picture is taken at $\mathrm{Ra}=6.8 \times 10^{8}$.

temperature fluctuations near the sidewall (top curve) are highly skewed toward one direction and the upward going spikes are associated with the rising warm plumes in the region. It is seen that the thermal plumes do not arrive randomly; rather, they arrive in groups with a welldefined frequency. In a recent experiment [14], we found that such an oscillation is caused by the alternating emission of cold and warm plumes between the upper and lower boundary layers. When the warm plumes move up in the sidewall region, they interact with the surrounding fluid through direct entrainment. As shown in Fig. 3(a), the resulting vertical velocity (middle curve) follows the temperature oscillation in the region. From the simultaneous velocity and temperature data, we obtain the instantaneous vertical flux (bottom curve), $j_{z}(\mathbf{r}, t)=$ $\left[v_{z}(\mathbf{r}, t) \delta T(\mathbf{r}, t)\right] H / \kappa \Delta T$, with $\left\langle j_{z}(\mathbf{r}, t)\right\rangle_{t}=J_{z}(\mathbf{r})$. In the calculation of $j_{z}(\mathbf{r}, t)$, we have used the convention that warm fluctuations $(\delta T>0)$ produce positive flux if their velocities are in the upward direction $\left(v_{z}>0\right)$.

Figure 3(b) shows the corresponding autocorrelation functions of $v_{z}, \delta T$, and $j_{z}$. It is seen that the instantaneous heat flux oscillates with the same frequency and phase as those of the temperature and velocity fluctuations. Figure 3 thus further confirms that the heat transport in turbulent convection is indeed carried out by the thermal plumes. Because of spatial average, the oscillatory behavior of the local heat flux is not observed in the global measurement of the Nusselt number.

Figure 4(a) shows the measured histograms of the instantaneous vertical flux $H\left(j_{z}\right)$ (triangles) and horizontal flux $H\left(j_{y}\right)$ (circles) near the sidewall. It is seen that fluctuations of the horizontal flux $j_{y}$ (out of the rotation plane

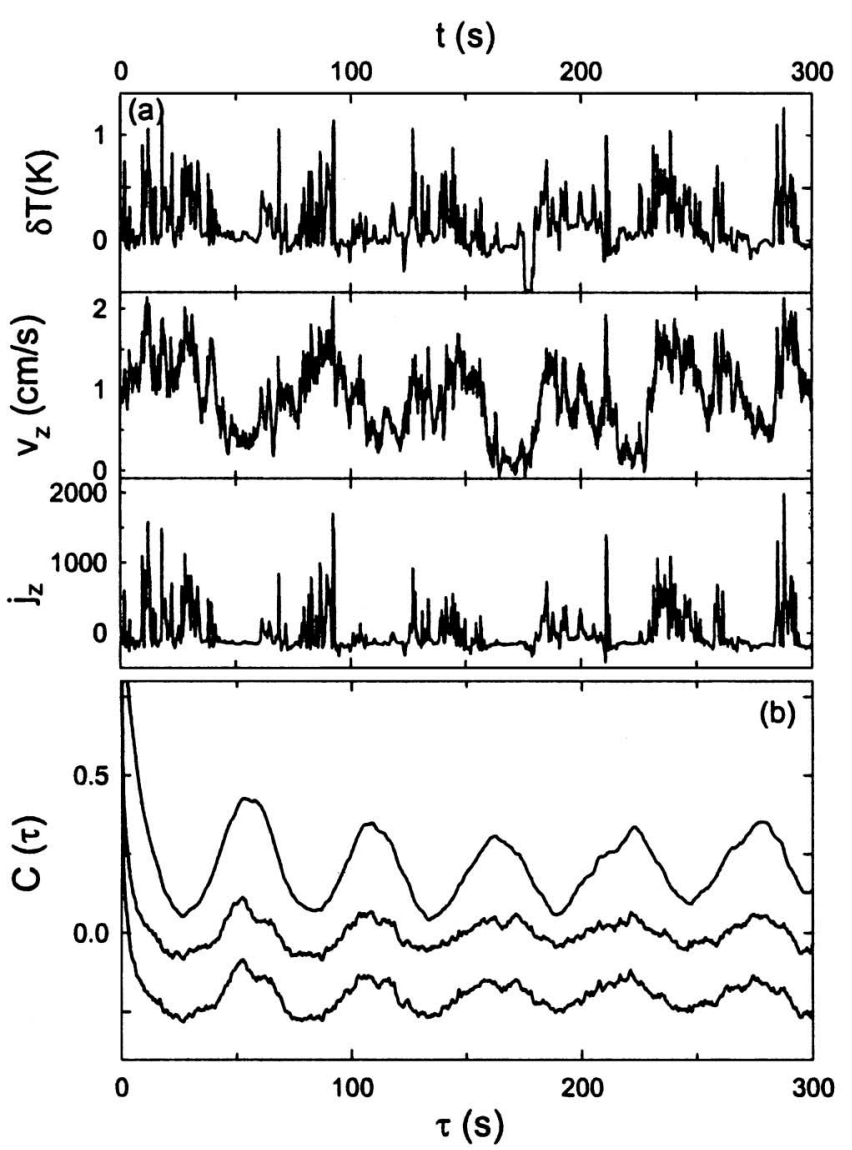

FIG. 3. (a) Time series measurements of the temperature fluctuation $\delta T(t)$ (top curve), vertical velocity $v_{z}(t)$ (middle curve), and instantaneous vertical flux $j_{z}(t)$ (bottom curve) near the sidewall. All the measurements are made at $\mathrm{Ra}=$ $3.6 \times 10^{9}$. (b) Corresponding autocorrelation functions $C(\tau)$ for $v_{z}(t)$ (top curve), $\delta T(t)$ (middle curve), and $j_{z}(t)$ (bottom curve). For clarity, the origin of the top and bottom curves is shifted by \pm 0.2 .

of LSC) are symmetric and their mean value is approximately zero. Fluctuations of the vertical flux $j_{z}$, on the other hand, are asymmetric with positive fluctuations being much larger than negative ones. The net gain of the positive fluctuations gives rise to a mean vertical heat flux. It is interesting to note that the negative flux fluctuations are approximately the same in both directions, whereas the positive flux fluctuations are much larger in the vertical direction parallel to gravity. Figure 4(a) reveals that the measured $j_{z}$ consists of two types of fluctuations: uncorrelated (passive) and correlated (active) fluctuations. The negative fluctuations are approximately isotropic and are produced by uncorrelated temperature and velocity fluctuations. Small positive fluctuations symmetric to the negative ones are also uncorrelated. These fluctuations do not contribute to the heat transport. Only the large positive fluctuations are correlated; they are produced primarily by the thermal plumes and make positive contributions to the mean flux. 


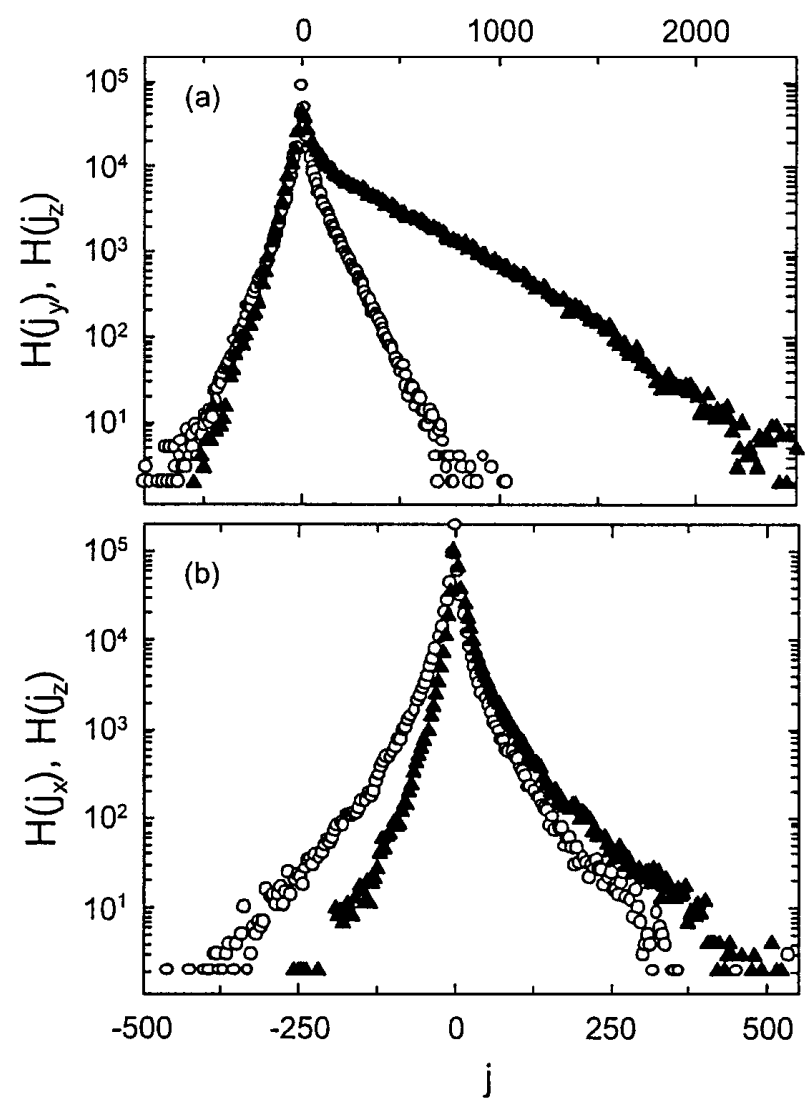

FIG. 4. (a) Measured heat flux histograms $H\left(j_{z}\right)$ (closed triangles) and $H\left(j_{y}\right)$ (open circles) near the sidewall $(9 \mathrm{~mm}$ from the sidewall). (b) Measured $H\left(j_{z}\right)$ (closed triangles) and $H\left(j_{x}\right)$ (open circles) at the cell center. All the measurements are made at $\mathrm{Ra}=2.6 \times 10^{9}$.

Figure 4(b) shows the measured $H\left(j_{z}\right)$ (triangles) and $H\left(j_{x}\right)$ (circles) at the center of the cell. Similar to the situation near the sidewall, fluctuations of the horizontal flux $j_{x}$ are symmetric with a mean value close to zero. Fluctuations of the vertical flux $j_{z}$, on the other hand, are asymmetric with negative fluctuations being smaller than positive ones. We believe that this asymmetry is generated by a small fraction of correlated signals (between temperature and velocity), which are produced by the remaining warm and cold plumes in the region. While temperature and velocity fluctuations are symmetric at the cell center, the correlated signals associated with the warm and cold plumes produce only positive flux in the vertical direction. As a result, the negative fluctuations are suppressed and the positive ones are enhanced when compared with the horizontal flux fluctuations. This is clearly shown in Fig. 4(b).

From Fig. 3(a) we find that the instantaneous vertical flux $j_{z}$ near the sidewall (bottom curve) is highly skewed toward one direction. Positive fluctuations are superposed on an average base line. These upward going spikes are associated with the rising warm plumes in the region. While the input heating power is constant (at a fixed $\mathrm{Ra}$ ) and uniform across the entire conducting surface, the release of this power to the convecting fluid $\left(\right.$ via $j_{z}$ ) is highly intermittent both in time and space. It is seen from Figs. 3(a) and 4(a) that there are some energetic thermal plumes, which carry an instantaneous heat flux as large as 2000. This value is approximately 25 times larger than the mean heating flux $(\mathrm{Nu} \simeq 80)$. It is also found that the measured $H\left(j_{z}\right)$ is highly non-Gaussian and exhibits an exponential-like tail.

In summary, the experiment clearly reveals that the heat transport in turbulent convection is carried out mainly by thermal plumes and that the local heat flux is highly intermittent both temporally and spatially. These features are associated with the plume dynamics near the thermal boundary layers and thus should not be sensitive to the large-scale flow structures in the cell. It is also found that the heat transport in the aspect-ratio-one cell takes place primarily along the cell periphery in the direction of the large-scale circulation. This result should apply to other small aspect-ratio cells, in which the largescale flow structure and the plume dynamics are found to be similar to those in the aspect-ratio-one cell [11]. It is certainly important to explore turbulent convection in large aspect-ratio cells and the present experiment is, in fact, the first step toward this direction.

We thank Siu Lam for his work in producing the shadowgraph and L. Kadanoff, D. Lohse, P. Constantin, and E. Ching for useful discussions. This work was supported in part by the NSF under Grant No. DMR0071323 (P.T.) and by the Hong Kong RGC under Grant No. CUHK4242/01P (K. Q. X.).

[1] E. Siggia, Annu. Rev. Fluid Mech. 26, 137 (1994).

[2] S. Grossmann and D. Lohse, J. Fluid Mech. 407, 27 (2000).

[3] L. P. Kadanoff, Phys. Today 54, No. 8, 34 (2001).

[4] B. Castaing et al., J. Fluid Mech. 204, 1 (1989).

[5] X. Chavanne et al., Phys. Rev. Lett. 79, 3648 (1997).

[6] J. J. Niemela et al., Nature (London) 404, 837 (2000).

[7] X. Xu et al., Phys. Rev. Lett. 84, 4357 (2000).

[8] G. Ahlers et al., Phys. Rev. Lett. 86, 3320 (2001).

[9] X.-Q. Xia et al., Phys. Rev. Lett. 88, 065501 (2002).

[10] Y.-B. Du and P. Tong, J. Fluid Mech. 407, 57 (2000).

[11] X.-L. Qiu and P. Tong, Phys. Rev. E 64, 036304 (2001); 66, 026308 (2002).

[12] The correlation length was obtained by varying the separation between the temperature and velocity probes. X.-D. Shang et al. (to be published).

[13] S.-L. Lui and K.-Q. Xia, Phys. Rev. E 57, 5494 (1998).

[14] X.-L. Qiu and P. Tong, Phys. Rev. Lett. 87, 094501 (2001).

[15] The convection cell is filled with a high Prandtl number fluid: dipropylene glycol $(\mathrm{Pr}=596)$, which has a large shadowgraphic contrast. Similar plume distribution is also observed in water, which has a much smaller shadowgraphic contrast. S. Lam, Master's thesis, The Chinese University of Hong Kong, 2000. 